\title{
On the development of a low-cost rigid borescopic fringe projection system
}

\author{
Jochen Schlobohm, Andreas Pösch, Markus Kästner, Eduard Reithmeier \\ Leibniz Universität Hannover, Mechanical Engineering, Institute of Measurement and \\ Automatic Control, Nienburger Str. 17, 30167 Hanover, Germany
}

\begin{abstract}
Examining the geometry of complex industrial free form objects, like a blade integrated disk (blisk) of a jet engine compressor, is currently subject to research. High measurement precision and speed are required and the complex geometry poses a challenge for state of the art measurement systems. In order to fulfill typical inspection requirements, the fringe projection methodology was adapted in this work to accomplish the task of fast and precise geometry examination. A low cost borescopic fringe projection system for 3D shape measurement based on consumer electronics combined with state of the art optics was developed. Nevertheless, it is able to provide measurement uncertainties comparable to professional systems. We are using a portable consumer LED-beamer, which we have modified to fit the optics of the borescope and a Raspberry Pi single-board computer with a 5 megapixel camera to capture the fringe patterns. With this setup and fringe projection algorithms, which have been developed by this institute over the last years, we were able to perform high quality measurements while still being suitable for a compact inspection system. Measurements with high point densities are possible even in narrow areas of parts with complex geometries like blisks. The measuring system and first measurement results will be presented at the conference.
\end{abstract}

Keywords: endoscope, borescope, measurement, optical measurement, fringe projection, blisk, projector

\section{INTRODUCTION}

Fringe projection allows fast and high precision 3D measurements of object surfaces. ${ }^{1}$ Especially the high lateral resolution or point density provides not only an objects macroscopic geometry, but makes it also possible to inspect an objects stochastic surface structure. This and the possibility to measure contact sensitive surfaces are just two advantages in comparison to current tactile coordinate measurement systems.

Bosch and NovaCam developed endoscopic low coherence interferometers, which are capable of measuring a single point with uncertainties as low as $1 \mu \mathrm{m}$. Though the measurement frequency may get as high as several $\mathrm{kHz}$, the overall measurement speed is still slow compared to fringe projection systems. Additionally, there is a need for a very precise position system to scan whole surfaces. With fringe projection each measurement consists of millions of data points which can be aligned very well using stitching algorithms.

The size of state of the art fringe projection systems makes it usually impossible to measure inside of complex geometries. As an example, blade integrated discs (blisks), as shown in Fig. 1, are difficult be measured with a fringe projection system, because it would not fit between two of the blades. Measuring the whole surface would imply to allow large angles between the surface normal and the line of sight. At those large angles, the reflectance of most surfaces is low which leads to lack of contrast of the projected fringes. Self shadowing of a rough surface may also decrease the measurement quality.

To circumwent those problems and to fullfill the task of full geometric insepection of complex geometries, we've developed a low-cost, rigid borescopic fringe projection system, mostly made our of of-the-shelf components.

Further author information: (Send correspondence to J. Schlobohm)

J. Schlobohm: E-mail: jochen.schlobohm@imr.uni-hannover.de, Telephone: +49 (0)511 7623236

Photonics, Devices, and Systems VI, edited by Pavel Tománek, Dagmar Senderáková, Petr Páta,

Proc. of SPIE Vol. 9450, 945009 - @ 2015 SPIE · CCC code: 0277-786X/15/\$18 · doi: 10.1117/12.2067310 


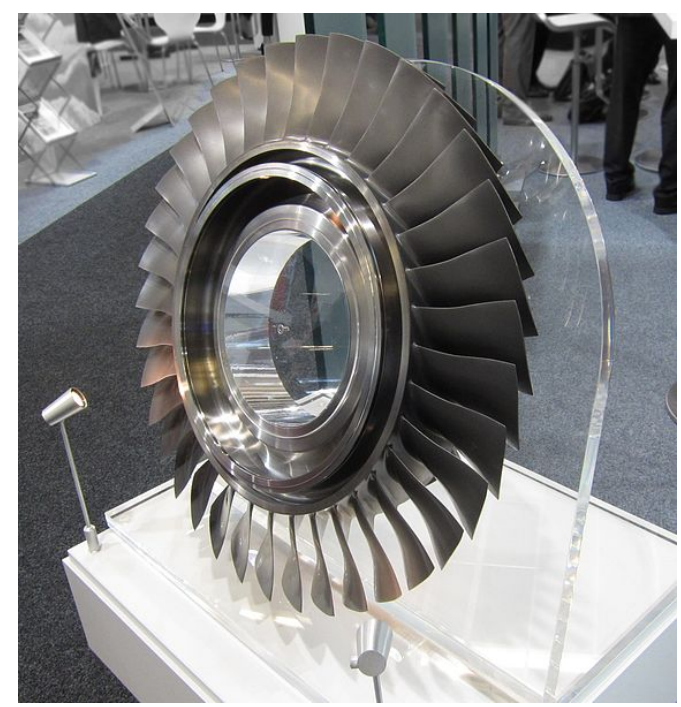

Figure 1: Example for a complex geometry: Blade integrated disc (Blisk). Source: Olivier Cleynen with creative common license $C C B Y$-SA 3.0

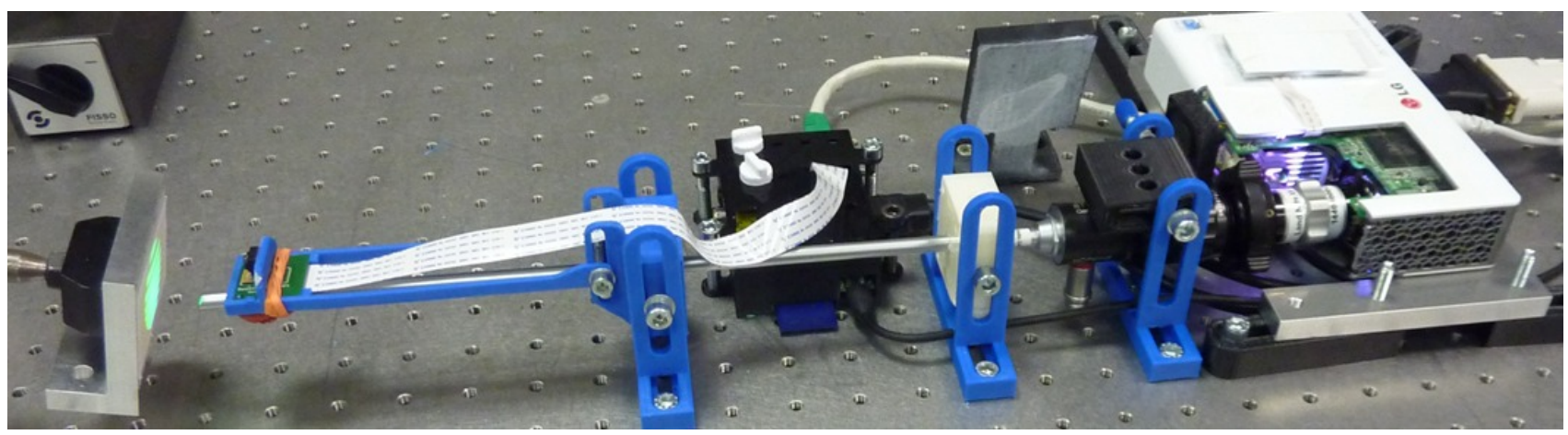

Figure 2: Photograph of the measuring setup: Camera and borescope head (left), borescope and adapter optics (middle), LED projector (right).

Although the measurement system is still in an early prototype stage, we are able to give first estimates of the measurement uncertainties.

\section{DESIGN OF THE MEASUREMENT SYSTEM}

\subsection{Hardware design}

As mentioned in the introduction, the prototype was build from of-the-shelf components. We're using a compact class LED based projector ( $L G P B 62 G$ ) with 720p HD-ready resolution, a 5 mega pixel mobile camera connected to a raspberry $P I^{2}$ single-board computer and a rigid boresope from Storz. ${ }^{3}$ The full setup is displayed in figures 2 and 3. In order to use the borescope for the projection of patterns, we removed the build in optics of the projector and added a c-mount adapter from storz. This adapter is usually used to combine a rigid borescope with an industrial c-mount camera. As a result we have a very high contrast of the projected patterns, as you can see in Fig. 4. The camera is typically used in cellphones. It is connected to the raspberry pi with the standard CSI (camera serial interface ${ }^{4}$ ).

The angle, in reference to the boresope shaft, of the line of sight of the borescope is $30^{\circ}$. The angle of the optical axis of the camera is $60^{\circ}$ which leads to an triangulation angle of approximately $30^{\circ}$, depending on the area of 


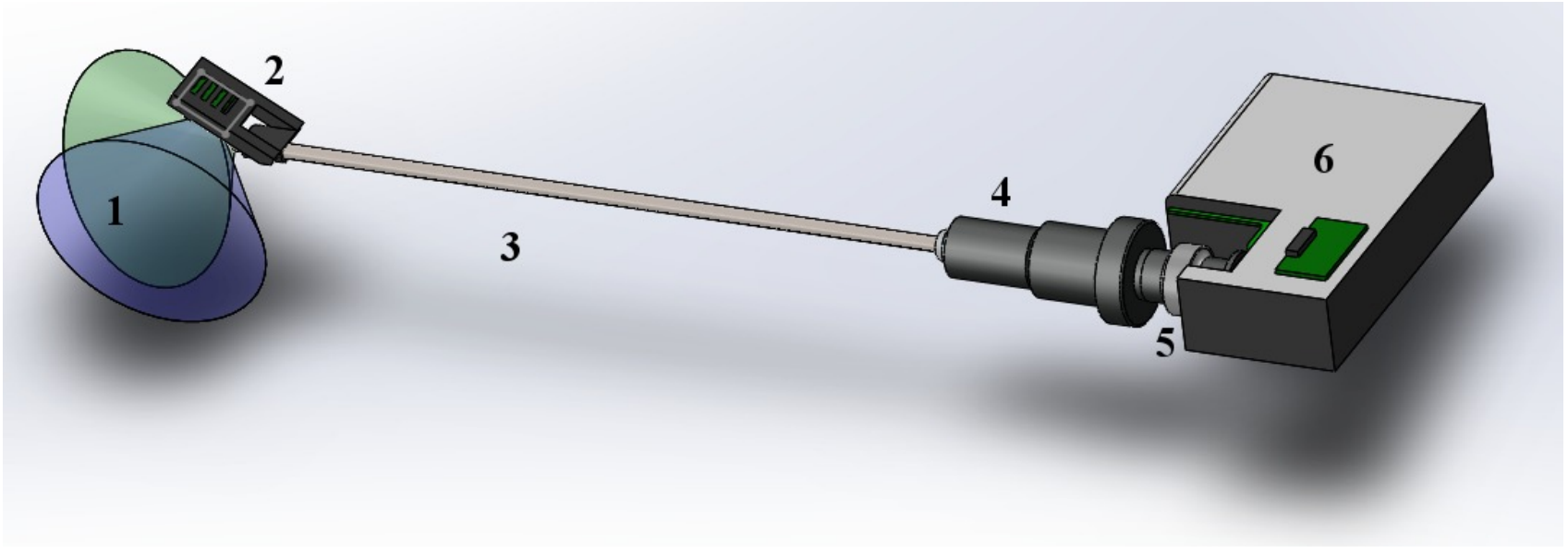

Figure 3: CAD model of the prototype. 1: fields of view, 2: camera, 3: borescope, 4: ocular, 5: c-mount adapter, 6: projector.

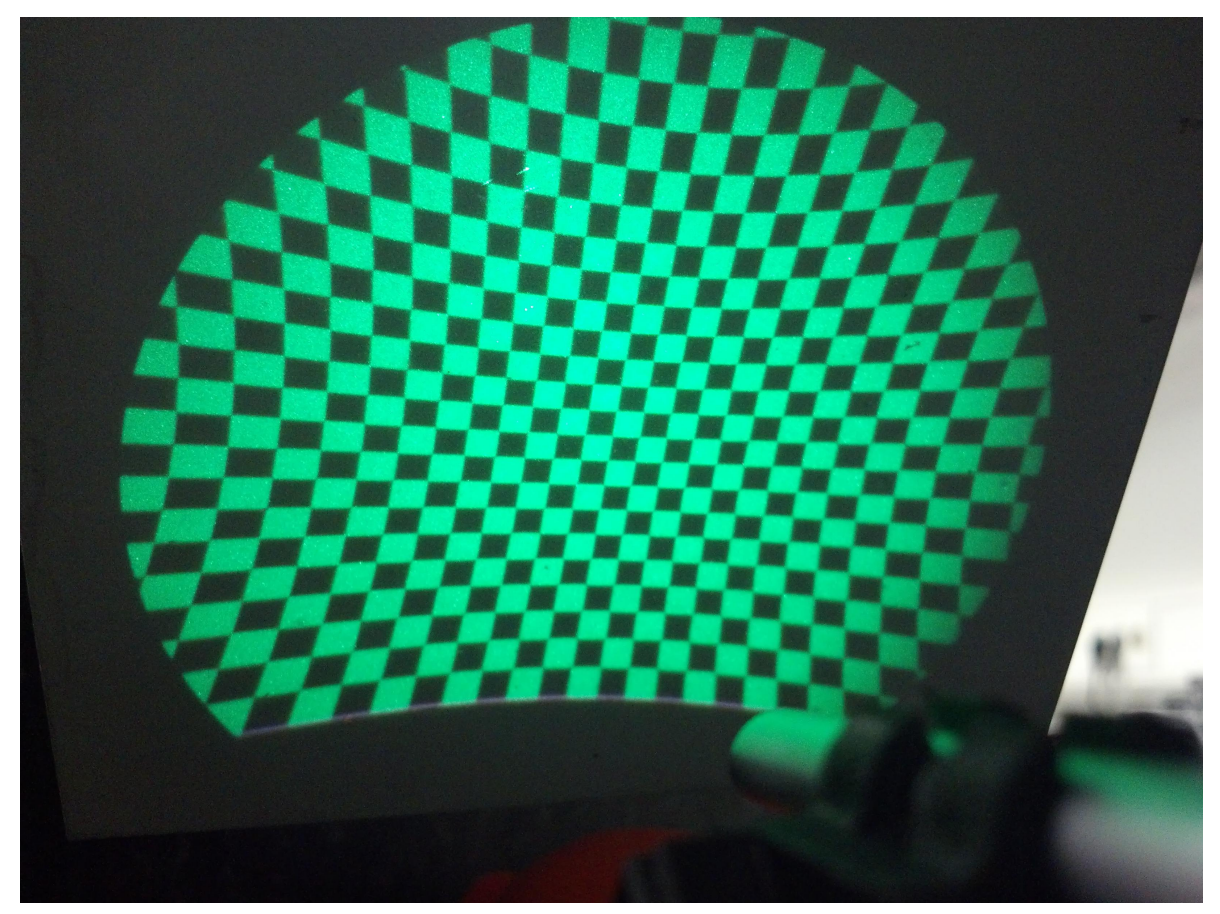

Figure 4: Photograph of a projected chessboard pattern on sanded aluminum using a borescope. 


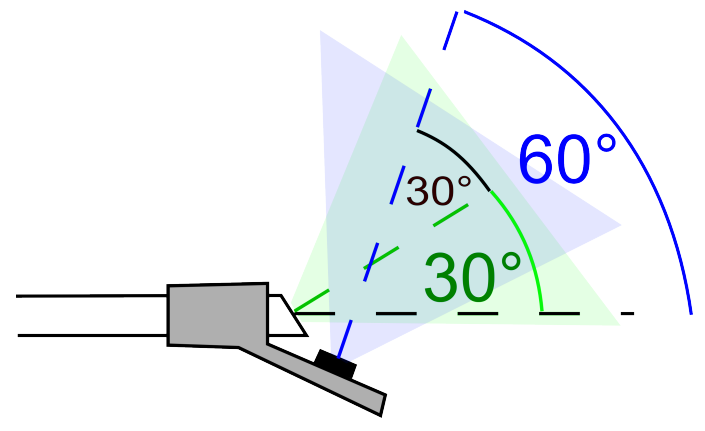

Figure 5: Sketch of the measurement head with the angles of view: Borescope shaft (white), camera (black), clip (grey), fields of view (light blue and green).

the field of measurement. The ideal angle between the borescope and the object's surface should therefore be around $\left./ 60^{\circ}+30^{\circ}\right) / 2=45^{\circ}$ as shown in Fig. 5. The angle of this borescope is fixed but borescopes with different angles (e.g. $\left.0^{\circ}, 60^{\circ}, 90^{\circ}\right)$ may be purchased. The camera is attached to the borescopes shaft with a plastic clip that we printed with a 3D printer. Using a different borescope angle would imply to print a new clip with a fitting angle. The camera is connected to the raspberry $p i$ with a flexible ribbon cable. Both, the projector and the raspberry pi are connected to a measurement computer to speed up pattern calculation and data processing.

\subsection{Software and data processing}

The images taken by the raspberry pi are transferred to the measurement computer using an open source rpc library. The fringe patterns are generated on a standard computer and transfered to the projector via HDMI. Projector and camera calibration are based on Zahng's method. ${ }^{5}$ Pösch et al. ${ }^{6}$ developed an advanced method to apply those algorithms to a camera-projector system. The camera has a color sensor and it's raw data is accessed. Current measurements use only the green pixels. Using multiple channels would make a more complex calibration necessary. This calibration would need to address the fact, that each color channels pixels are shifted according to the bayer pattern used. As usual with bayer patterns, there are as many green pixels as red and blue together, so that green pixels cover half of the chip area. Every second row of green pixels is shifted by one pixel width. In order to achieve a regular chessboard like pattern, the values of two green pixels each are averaged. The camera sensor has $(2592 \times 1944) p x=5038848 p x$. With every second pixel being green and averaging the effective resolution is $(1296 \times 972) p x$.

The light path for projecting the patterns consists of several optical systems, which causes complex distortion effects. The optical systems, especially the borescope relative to the projector, are aligned by hand. This leads to tangential distortion. Therefore we extended the distortion model with parameters for tangential distortion to improve the measurement quality.

\section{RESULTS}

To get a first impression on measurement uncertainties, we have measured two primitive objects: a sanded aluminum plane and a calibration ball with a diameter of $30 \mathrm{~mm}$. We assume those objects to have a perfect geometry in reference to the measurement precision of our system. Figure 6 shows the measurement of the plane with the distance of each point to a fitted plane. Most points distance is in the range of $+-30 \mu m$. As can be seen, the corners are bend away from the camera which is an indicator of lens distortion. Lens distortion is usually bigger, the further a pixel is away from the image center.

Figure 7 shows the measurement of the calibration ball. The results are similar to the measurement of the plane. The ripples in the mid section are created due to saturation of the camera sensor. The small bumps in the top 


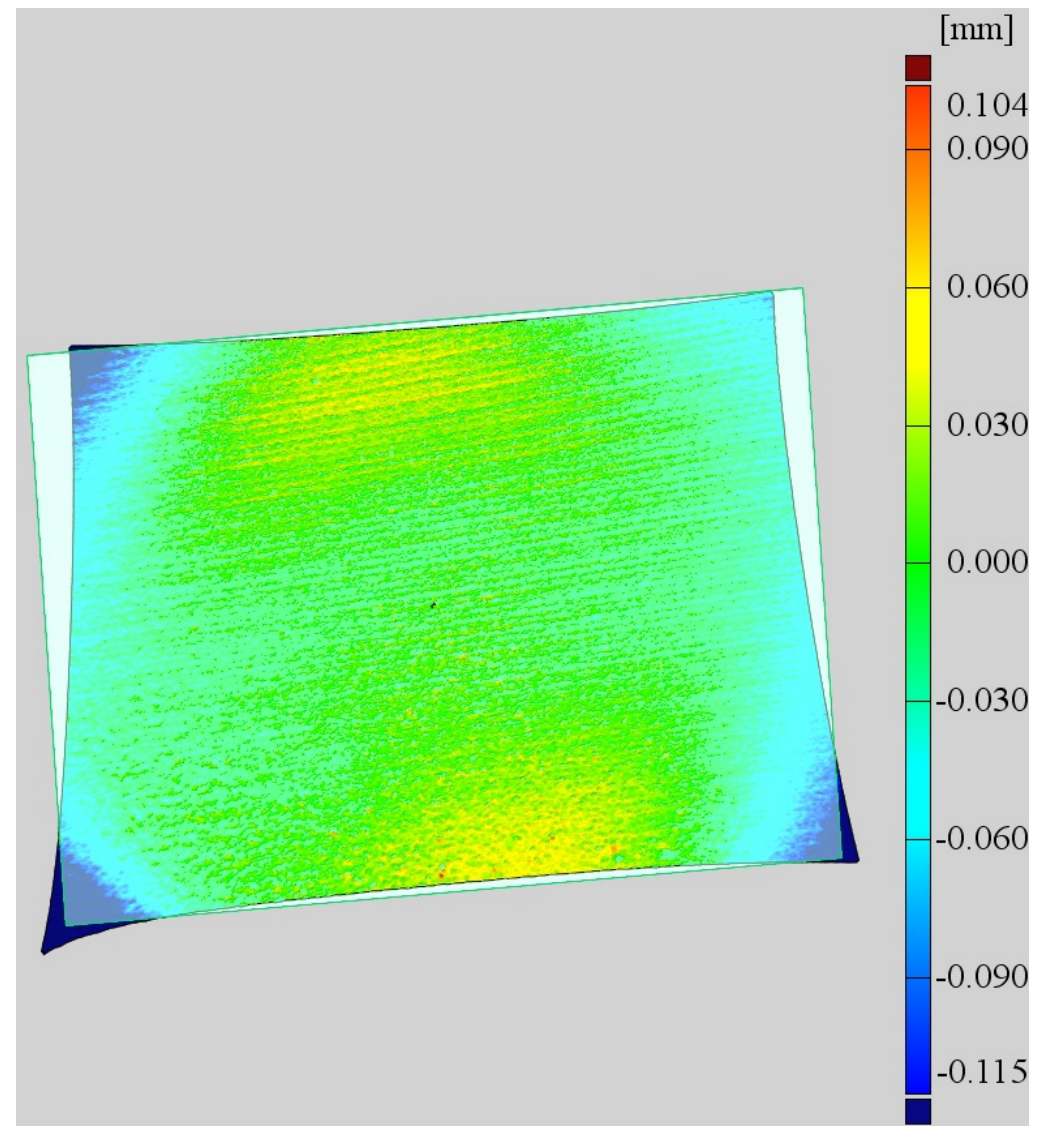

Figure 6: Measurement of a calibration ball with 30mm diameter.

and the bottom of the image were caused by dust particles which we missed to remove for the measurement.

\section{SUMMARY AND PROSPECTS}

We were able to develop a low-cost borescopic measurement system, which is capable of measuring inside complex geometries. Using cost efficient standard components saved us time and money in the development process. State of the art calibration techniques where used to circumvent some disadvantages of those components. We where able to take some good quality measurements, although a full uncertainty characterization was not done yet. The system is still in an early development stage. The calibration of the system should be improved to address the bend corners seen in Fig. 6. Also high dynamic range techniques could be applied to address the ripples in the mid section of the ball measurement in figure 7 .

New hardware, like a smaller camera, will allow us to construct an even smaller measurement head. Another step will be to exchange the small camera on the tip with a standard industrial camera along with a second borescope for image acquisition. With that setup we hope to improve the effective resolution and get a better signal to noise ratio. Borescopes with different angles of view will be used and tested.

\section{ACKNOWLEDGMENTS}

We would like to thank the German Research Foundation (DFG) for funding this project within the Collaborate Research Center (SFB) 871 regeneration of complex capital goods (http://www.sfb871.de). 


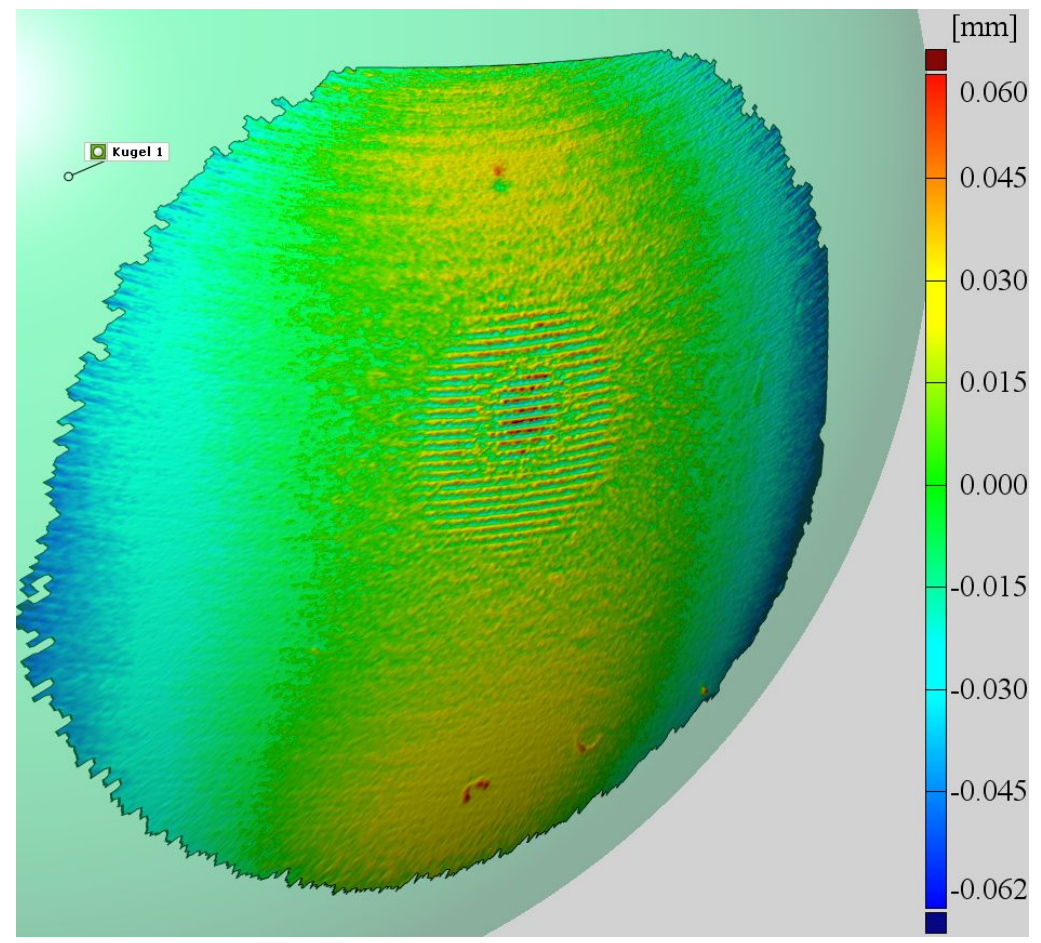

Figure 7: Measurement of a calibration ball with 30mm diameter.

\section{REFERENCES}

[1] Kästner, M., Optische Geometrieüberprüfung präzisionsgeschmiedeter Hochleistungsbauteile, PhD thesis, Leibniz Universität Hannover (2008).

[2] "Raspberry pi project homepage." online (07 2014). http://www.raspberrypi.org/.

[3] Storz, "Messysteme und dokumentation." online (07 2014). https://www.karlstorz.com/cps/rde/xchg/karlstorzen/hs.xsl/15522.htm.

[4] mipi alliance, "Camera interface specifications." online (07 2014). http://mipi.org/specifications/camerainterface.

[5] Zhang, Z., "A flexible new technique for camera calibration," IEEE Transactions on Pattern Analysis and Machine Intelligence 22(11), 1330-1334 (2000).

[6] Pösch, A., Vynnyk, T., and Reithmeier, E., "Fast detection of geometry defects on free form surfaces using inverse fringe projection," Journal of the CMSC 8(1), 10-13 (2013). 